\title{
Energisch, heiter und gelassen
}

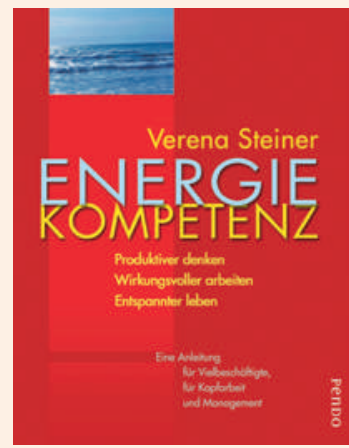

Verena Steiner Energiekompetenz Produktiver denken Wirkungsvoller arbeiten Entspannter leben München: Pendo; 2011 284 Seiten, $28.90 \mathrm{CHF}$ ISBN 978-3-86612-030-3

Korrespondenz:

Dr. med. Bernhard Gurtner

Eggstrasse 76

CH-8620 Wetzikon

gurtner.bernhard[at]bluewin.ch
Ein 2005 erstmals veröffentlichtes Fachbuch, das schon in der 8. Auflage angeboten wird, benötigt keine zusätzliche Empfehlung. Es verdient jedoch die Beachtung unserer Berufsgruppe, die für einmal aufmunternde Ratschläge selbst befolgen könnte, die sie sonst gerne nur ihren Patienten erteilt. Ärzte und Ärztinnen klagen ja über zunehmende Unlust und sollen laut Umfragen bis zu 50\% von Burnout bedroht oder betroffen sein. Da lässt sich manches dagegen tun, was die erfahrene Autorin überzeugend darlegt. Mit Energie-Kompetenz ist aber nicht etwa gemeint, letzte Reserven aus sich herauszuholen, vielmehr wird mit biologisch und psychologisch fundierten praktischen Hinweisen gezeigt, wie entspannteres Leben dazu verhilft, immer wieder neuen Schwung und Freude für die Bewältigung persönlicher und gesellschaftlicher Anforderungen zu gewinnen.

Verena Steiner steht der Medizin nicht fern, weil sie nach Studium der Biochemie an der Universität Basel als Forscherin und Managerin in der Pharmaindustrie tätig war. Von 1994-2001 wirkte sie an der ETH Zürich, wo sie vielbesuchte Kurse für Lern-, Denk- und Arbeitsstrategien einführte. Während eines Sabbaticals 1999 an der Harvard University konzipierte sie das Buch «Exploratives Lernen», das sich mit seinen Anleitungen zu lustbetontem Wissenserwerb als Bestseller erwies (12. akt. Auflage 2013) und der beliebten Referentin eine Gastprofessur in Wien eintrug. Frau Steiner ist mit einem Internisten verheiratet und hat so direkten Einblick in die Anforderungen und Probleme des ärztlichen Alltags.

Als passionierte Läuferin mutet Verena Steiner ihren Lesern einen 270 Seiten langen Parcours zu, den sie aber sehr abwechlungsreich gestaltet hat. Nach anspruchsvollen gedanklichen Steigungen kann man sich auf flacherem Gelände geistig erholen. Einige Aussichtspunkte werden mit didaktischer Absicht mehrmals angelaufen, die Eindrücke bleiben dank Repetition besser haften. Am Weg stehen fast 150 Merktafeln, auf denen Dichter und Philosophen aus verschiedenen Perioden und Kulturkreisen, sowie zeitgenössische Künstler, Sportler, Politiker und Manager mit Zitaten zu Erkenntnisgewinnen verhelfen. Als Randbemerkungen liest man europäische und asiatische Sprichwörter, Zen-Weisheiten und je einen Merksatz aus Bibel und Koran. Schön, dass der Abenteurer und Psychiater Bertrand Piccard mit fünf Zitaten ebenso oft vertreten ist wie der Geheimrat Johann Wolfgang von Goethe, der Wirtschaftspädagoge Karlheinz Geissler und der buddhistische Mönch Thich Nhat Hanh.

Die in drei Hauptabschnitte aufgeteilten zehn Kapitel sind klar strukturiert und flüssig geschrieben.
Der erste Teil hilft zur besseren Selbstbeobachtung der inneren Rhythmen. Studienergebnisse der Chronobiologie werden umgesetzt in Anweisungen, damit Morgen- oder Abendtypen ihre Primetime und die Up- oder Downphasen optimal nutzen können, - so weit es eben die Lebensumstände erlauben, die heute allen einen Non-Stop-Modus aufzwingen wollen. Koffeinhaltige Getränke als Aufputschmittel würden zweckmässiger durch kurze Nickerchen ersetzt, welche die Energien wieder aufladen.

Gut schlafen ist lernbar. Habituelle Schlafstörungen können ohne Medikamente beseitigt werden, wenn man die klugen Ratschläge der Autorin befolgt. Sie empfiehlt einen liebevollen Umgang mit sich selbst, wie mit einem jungen Hund, dem man Gewohnheiten sanft, aber mit unerbittlicher Konsequenz beibringen muss. Spätes Aufstehen am Samstag und Sonntag könne das Schlafmanko der zuvor im Ausgang verbrachten Nächte nur scheinbar kompensieren, weil die innere Uhr durch zweimaliges Langschlafen so umgestellt wird, dass am Montagmorgen das bekannte Jetlag-Gefühl verbleibt. Das Wochenende sollte wieder als geruhsamere Erholungszone mit Sinnierpausen kultiviert werden.

Im Teil II wird die Selbstwahrnehmung der jeweiligen Energie- und Anspannungsphasen anhand einer Matrix geschult, die goldene Zeit des völlig entspannten Alpha-Zustandes geschildert und erklärt, wie man mit seinen Stimmungsschwankungen umgeht. Auch in diesem theoretisch ausführlichen Kapitel sind praktische Anregungen eingestreut.

Teil III will aufzeigen, wie man neue Energien gewinnen kann durch eingeübte muskuläre und mentale Entspannung, richtig dosierte Erholung und regelmässigen Sport ohne Stress und Strebertum. Mit allen Sinnen können die kleinen Freuden im Alltag und die Wunder der Natur wahrgenommen werden. Auch einige altüberlieferte Tugenden wie Selbstüberwindung, Konsumverzicht oder Pünktlichkeit werden mit konkreten Beispielen als Energiespender in Erinnerung gebracht. Moderner tönt der Begriff Commitment als Ausdruck eines vorbehaltlosen und herzhaften Einsatzes für eine Aufgabe.

Wer nun glaubt, dass ihn die Thematik dieses Buches nicht betrifft, müsste sich fragen, ob er nicht zu jenen chronisch angespannten Menschen gehört, die sich ihres Stresses gar nicht mehr bewusst sind, ihn als normalen Dauerzustand betrachten und keinen Grund sehen, daran etwas zu ändern - bis es zu spät ist. Dem könnte das anregende Buch von Verena Steiner entgegenwirken und Stressgefährdeten helfen, wieder besser zu sich selber zu finden und durch Entspannung neue Energien freizusetzen. 